\title{
Downstream Propagation and Remote Dissipation of Internal Waves in the Southern Ocean
}

\author{
KAIWEN ZHENG \\ Physical Oceanography Laboratory/Collaborative Innovation Center of Marine Science and Technology, Ocean University of \\ China, and Qingdao National Laboratory for Marine Science and Technology, Qingdao, China, and Institute for \\ Marine and Antarctic Studies, University of Tasmania, Hobart, Tasmania, Australia \\ MAXIM NIKURASHIN \\ Institute for Marine and Antarctic Studies, University of Tasmania, Hobart, Tasmania, and Centre of Excellence for \\ Climate Extremes, Sydney, New South Wales, Australia
}

(Manuscript received 22 June 2018, in final form 22 April 2019)

\begin{abstract}
Recent microstructure observations in the Southern Ocean report enhanced internal gravity waves and turbulence in the frontal regions of the Antarctic Circumpolar Current extending a kilometer above rough bottom topography. Idealized numerical simulations and linear theory show that geostrophic flows impinging on rough small-scale topography are very effective generators of internal waves and estimate vigorous wave radiation, breaking, and turbulence within a kilometer above bottom. However, both idealized simulations and linear theory assume periodic and spatially uniform topography and tend to overestimate the observed levels of turbulent energy dissipation locally at the generation sites. In this study, we explore the downstream evolution and remote dissipation of internal waves generated by geostrophic flows using a series of numerical, realistic topography simulations and parameters typical of Drake Passage. The results show that significant levels of internal wave kinetic energy and energy dissipation are present downstream of the rough topography, internal wave generation site. About $30 \%-40 \%$ of the energy dissipation occurs locally over the rough topography region, where internal waves are generated. The rest of the energy dissipation takes place remotely and decays downstream of the generation site with an $e$-folding length scale of up to $20-30 \mathrm{~km}$. The model we use is two-dimensional with enhanced viscosity coefficients, and hence it can result in the underestimation of the remote wave dissipation and its decay length scale. The implications of our results for turbulent energy dissipation observations and mixing parameterizations are discussed.
\end{abstract}

\section{Introduction}

Recent observations report enhanced internal wave kinetic energy and turbulent energy dissipation in the bottom kilometer in the Southern Ocean (e.g., Naveira Garabato et al. 2004; St. Laurent et al. 2012; Waterman et al. 2013; Sheen et al. 2013). This enhancement corresponds to the fronts of the Antarctic Circumpolar Current (ACC) characterized by strong bottom flows. When a bottom flow impinges on rough, small-scale $[O(1-10) \mathrm{km}]$ topography, it generates internal lee waves, which radiate away and break, thus sustaining the observed turbulence and mixing (e.g., Nikurashin and Ferrari 2010a,b). This mixing drives the transformation of water masses and hence contributes to

\footnotetext{
Corresponding author: Kaiwen Zheng, keviniskg@sina.com
}

the maintenance of the deep ocean stratification and the meridional overturning circulation (e.g., Munk and Wunsch 1998; Nikurashin and Ferrari 2013; de Lavergne et al. 2016).

The wind power input into geostrophic flows in the ocean is estimated to be of $O(1) \mathrm{TW}\left(1 \mathrm{TW}=10^{12} \mathrm{~W}\right.$; e.g., Munk and Wunsch 1998) with $80 \%$ of this input taking place in the Southern Ocean (e.g., Roquet et al. 2011). Part of this energy is converted into lee waves when geostrophic flows impinge on rough topography at the ocean floor. Previous theoretical studies have estimated that the global energy conversion into lee waves is in the range from 0.2 to $0.75 \mathrm{TW}$, dominated also by the Southern Ocean (Nikurashin and Ferrari 2011; Scott et al. 2011; Wright et al. 2014). Using these global estimates as an energy source for mixing, a parameterization of lee-wave-driven mixing has been implemented in 
ocean and climate models and shown to impact the global ocean stratification and overturning circulation (e.g., Melet et al. 2014, 2015). However, these global estimates and parameterizations rely on a linear theory of lee wave generation in a stratified flow interacting with rough bottom topography (e.g., Bell 1975a,b) and hence assume small-amplitude and horizontally uniform topography and local lee wave energy dissipation.

While the observed turbulent energy dissipation in the Southern Ocean is believed to be sustained mainly by the topographically generated internal waves, significant differences between the theoretical estimates of the energy conversion into lee waves and direct turbulent energy dissipation measurements have been recently reported (St. Laurent et al. 2012; Waterman et al. 2013; Sheen et al. 2013; Waterhouse et al. 2014). In the Drake Passage region, the linear theory estimates that the vertical energy flux associated with bottom internal wave generation averaged over the region is $14 \mathrm{~mW} \mathrm{~m}^{-2}$. However, only $10 \%-30 \%$ of the estimated energy flux was observed to dissipate within the bottom kilometer in the Drake Passage region as part of the Diapycnal and Isopycnal Mixing Experiment in the Southern Ocean (DIMES; St. Laurent et al. 2012; Sheen et al. 2013). Similarly, observations collected from the Southern Ocean Finestructure (SOFine) experiment in the region of the northern Kerguelen Plateau suggested that the observed deep dissipation accounts for only a small part (less than $20 \%$ ) of the estimated lee wave energy flux (Waterman et al. 2013).

Recent studies have tested some of the assumptions of the linear theory and mixing parameterization and suggested several mechanisms that could contribute to the reported difference. One interpretation is that finite-amplitude bottom topography effects will suppress the generation of internal lee waves (Nikurashin et al. 2014). When the topographic steepness parameter, a ratio of the topographic slope to internal wave slope, exceeds a critical value of 0.4 , a certain fraction of the mean flow will be blocked and split by topography. In this limit, the effective amplitudes of the radiating lee waves, and therefore the magnitude of lee wave energy flux and corresponding energy dissipation, are substantially reduced. This effect can be particularly strong in regions characterized by high steepness parameter values such as Drake Passage in the Southern Ocean. On the other hand, in the SOFine region characterized by below critical steepness parameter values, other mechanisms may be at play. A possible explanation is that part of the lee wave energy can be absorbed by the mean flow through the wave-mean flow interaction, when the mean flow is characterized by a large near-bottom shear (Waterman et al. 2014). Finally, Trossman et al. (2015) suggested that anisotropy in abyssal hill topography and the relative orientation of the bottom flow with respect to this anisotropy, not taken into account in theoretical estimates, may also contribute to local overestimation of the wave energy radiation.

Motivated by this reported difference between the energy conversion into lee waves and local energy dissipation, in this study, we test the assumption of uniform (periodic) topography made both in linear theory and previous idealized numerical simulations and explore a hypothesis that lee waves can dissipate their energy remotely, downstream of the rough topography, wave generation site. Waterman et al. (2013) showed depthintegrated turbulent energy dissipation station by station and its relation to local magnitudes of the bottom flow and topographic roughness and found no explicit relationship between them: there are sites with large topographic roughness and strong background flow, but weak energy dissipation, and vice versa. Also, relatively flat topography regions with more turbulent energy dissipation than estimated from theory have been reported by Waterhouse et al. (2014), suggesting that lee waves propagate horizontally some distance away from their generation sites before dissipating. More direct evidence of the remote internal wave dissipation in the Southern Ocean is reported in a series of internal wave field observations north of the Kerguelen Plateau (Meyer et al. 2016). Based on float measurements, 46 internal waves from different sources have been identified. Comparing the propagation and dissipation time scales, about $1 / 3$ of the internal waves were found to dissipate their energy locally, while other waves propagated away from the Kerguelen Plateau and contributed to remote dissipation. In this study, we investigate the propagation away from the generation site and remote dissipation of internal waves generated by a mean flow impinging on rough bottom topography in the Southern Ocean using numerical simulations.

The paper is structured as follows. Section 2 describes the abyssal hill topography and flow characteristics in the region of Drake Passage and the model configuration used in this work. In this section, the flow decomposition method and diagnostics used for the analysis are also presented. In section 3, the results of a series of numerical simulations focusing on the downstream evolution and remote dissipation of internal waves generated by a uniform mean flow impinging on rough bottom topography are discussed. Conclusions and implications for the ocean observations and mixing parameterizations are presented in section 4 . 


\section{Methods}

We carry out a series of two-dimensional (2D) numerical simulations of the flow response to a uniform, steady geostrophic current impinging on rough bottom topography. Our reference simulation is based on realistic, multiscale abyssal hill topography and flow characteristics observed in the Drake Passage region of the Southern Ocean. Drake Passage is a region with abundant direct observations and characterized by high rates of turbulent energy dissipation and mixing. Below, we describe the flow characteristics in Drake Passage, model configuration, and a series of perturbation experiments that we run to investigate the mechanisms leading to the nonlocal wave dissipation. The flow decomposition and energy budget diagnostics are also discussed in this section.

\section{a. Characteristics of the geostrophic flow and bottom topography in Drake Passage}

Drake Passage is characterized by strong bottom flows and rough topographic features and, as a result, by high rates of abyssal turbulence and mixing (e.g., St. Laurent et al. 2012). It is also a region of rich observations of geostrophic velocity and stratification, internal waves, turbulence, and abyssal hill topography, and thus is a perfect site to study the lee wave driven mixing (e.g., Naveira Garabato et al. 2004; Sheen et al. 2013). In this study, we follow Nikurashin and Ferrari (2010b) and Nikurashin et al. (2014) and use parameters typical of Drake Passage for our reference simulation, which we briefly describe below. The reader is referred to Nikurashin and Ferrari (2010b) and Nikurashin et al. (2014) for a more detailed description.

The geostrophic flow in Drake Passage is dominated by strong ACC fronts, which collectively carry a large volume of water through the passage into the Scotia Sea. Instantaneous measurements from full-depth lowered acoustic Doppler current profilers (LADCP) and hydrography observations have shown large nearbottom currents with velocities reaching up to $10-20 \mathrm{~cm} \mathrm{~s}^{-1}$ above topography (Cunningham et al. 2003). Year-longmean near-bottom velocities calculated from measurements at different sites within the passage are also found to be over $10 \mathrm{~cm} \mathrm{~s}^{-1}$ in most parts of the region (Kunze et al. 2006; Chereskin et al. 2009). The mean current varies slowly with depth and remains essentially constant until it reach the thermocline. The observed bottom stratification varies across Drake Passage from $0.5 \times 10^{-3} \mathrm{~s}^{-1}$ in the south to $1 \times 10^{-3} \mathrm{~s}^{-1}$ in the north. Stratification in the Polar Front of the ACC, which dominates lee wave generation (Nikurashin and Ferrari 2010b), is close to $1 \times 10^{-3} \mathrm{~s}^{-1}$ in the bottom kilometer and slowly increases toward the surface. This study extends the reference Drake Passage simulations detailed in Nikurashin et al. (2014) to investigate the nonlocal dissipation of lee wave energy. A vertically uniform mean flow of $10 \mathrm{~cm} \mathrm{~s}^{-1}$ and stratification of $10^{-3} \mathrm{~s}^{-1}$ are imposed in the simulation. In the ocean, the interaction between vertically propagating internal waves and variations in flow velocity and stratification can lead to wave-mean and wave-wave interactions and subsequent energy dissipation (e.g., Gayen et al. 2013). In this study, however, we focus on the downstream propagation of lee wave energy and hence use a constant stratification and flow velocity to isolate the mechanisms and simplify interpretation. While the mean flow in our model setup represents all subinertial flows, the downstream propagation and remote dissipation of internal lee waves is more relevant to the wave generation by the ACC fronts than eddies. Yang et al. (2018) have recently estimated that $30 \%$ of lee waves in the Southern Ocean is generated by time-mean flows and $70 \%$ by transient eddies. In addition, the DIMES and SOFine results (e.g., St. Laurent et al. 2012; Sheen et al. 2013; Waterman et al. 2013) show a strong correspondence between the location of the ACC fronts and enhanced turbulent energy dissipation.

In comparison to other regions of the Southern Ocean, the bottom topography in Drake Passage is relatively rough and dominated by abyssal hills with 1-100-km horizontal scales (e.g., Nikurashin and Ferrari 2011). Lee waves are primarily generated from topographic scales from 1 to $10 \mathrm{~km}$ with the lee wave vertical energy flux dominated by $2-3-\mathrm{km}$ scale abyssal hills. To characterize the abyssal hill topography in Drake Passage, following Nikurashin et al. (2014), we used a onedimensional (1D) topography spectrum obtained by integrating the 2D spectrum for abyssal hills proposed by Goff and Jordan (1988),

$$
P_{2 \mathrm{D}}(k, l)=\frac{2 \pi H^{2}(\mu-2)}{k_{0} l_{0}}\left(1+\frac{k^{2}}{k_{0}^{2}}+\frac{l^{2}}{l_{0}^{2}}\right)^{-\mu / 2},
$$

along the wavenumber $l$, where the representative parameters of the Drake Passage region are the characteristic wavenumbers $k_{0}=2.3 \times 10^{-4} \mathrm{~m}^{-1}$ and $l_{0}=1.3 \times 10^{-4} \mathrm{~m}^{-1}$, root-mean-square height of abyssal hills $H=350 \mathrm{~m}$, and the high-wavenumber spectral slope $\mu=3.5$. This spectrum describes the variance of the topographic scales which account for most of the topographic lee wave generation. Synthetic topography (Fig. 1) is then computed as a sum of Fourier modes with amplitudes given by the $1 \mathrm{D}$ spectrum and random phases. This topography representation is the 

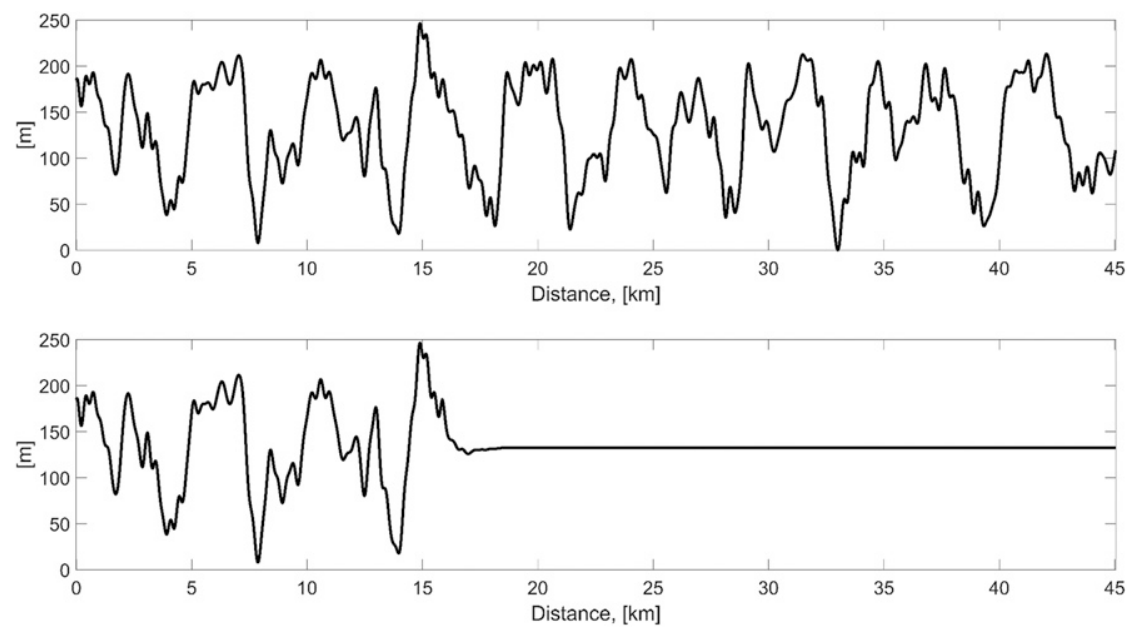

FIG. 1. Bottom topography used in (top) the reference experiment and (bottom) the rough and flat topography experiment.

same as what was used in previous internal wave modeling studies (Nikurashin and Ferrari 2010b; Nikurashin et al. 2014; Richet et al. 2017).

\section{b. Model configuration}

We employ the nonhydrostatic configuration of the Massachusetts Institute of Technology general circulation model (MITgcm; Marshall et al. 1997). This model has been widely used for studies of topographic internal wave generation in the ocean before (e.g., Nikurashin and Ferrari 2010b; Nikurashin et al. 2014; Legg and Klymak 2008; Richet et al. 2017; Klymak 2018). To represent the flow-topography interaction in Drake Passage in our reference simulation, we use a numerical setup similar to that in Nikurashin et al. (2014).
The domain used in all our simulations is $2 \mathrm{D}$ (Fig. 2) with a resolution of $5 \mathrm{~m}$ both in horizontal and vertical. The horizontal length of the domain is $45 \mathrm{~km}$. There are 4-km-thick sponge layers on each side of the domain, where velocities and stratification are restored back to unperturbed uniform values with the restoring time scale of $1 \mathrm{~h}$. The total depth is $3 \mathrm{~km}$. A depthindependent mean flow $U_{0}=0.1 \mathrm{~m} \mathrm{~s}^{-1}$ is forced by adding a body force to the meridional momentum equation representing a barotropic pressure gradient, which balances the mean flow geostrophically at all depths. A uniform stratification $N=10^{-3} \mathrm{~s}^{-1}$ and a Coriolis frequency of $f=-1.2 \times 10^{-4} \mathrm{~s}^{-1}$ are used. The Laplacian horizontal and vertical viscosity and diffusivity are set to $A_{h}=A_{v}=5 \times 10^{-3} \mathrm{~m}^{2} \mathrm{~s}^{-1}$ and

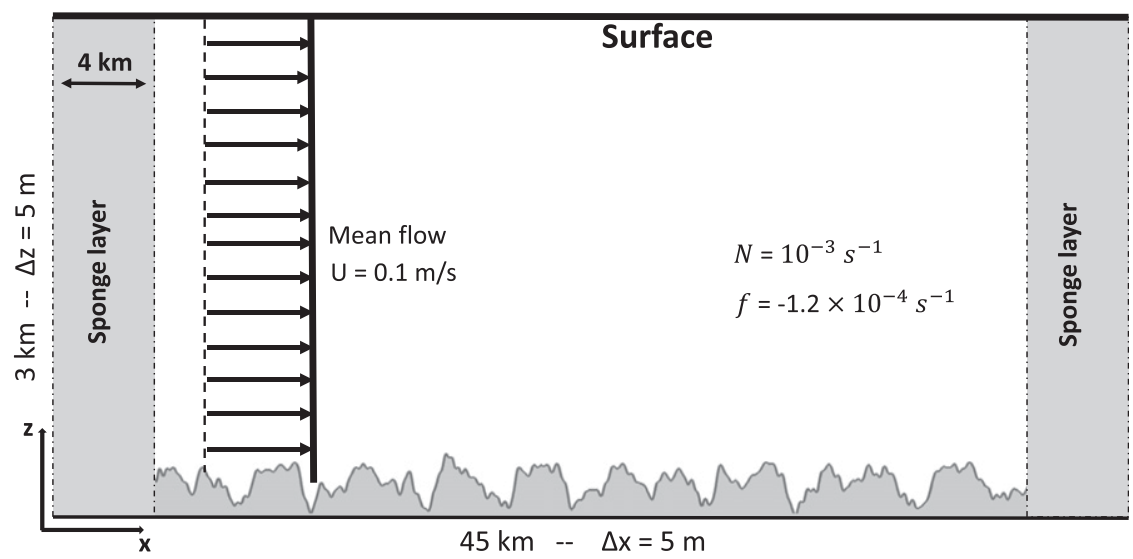

FIG. 2. Schematic of the model setup. The depth-independent mean flow interacts with realistic bottom topography in a stratified ( $N$ constant) and rotating ( $f$ constant) fluid. The gray, shaded regions on each side of the domain indicate sponge layers. There is a free surface at the top of the model domain in the reference experiment, so that waves can reflect when they get to the upper boundary. 
$\kappa_{h}=\kappa_{v}=10^{-5} \mathrm{~m}^{2} \mathrm{~s}^{-1}$, respectively. All model experiments are initialized from the state of rest and run for 10 days. The flow reaches a statistical steady state after about 5 days (Fig. 3 ) and the last 5 days are used for the analysis.

Although the model configuration is $2 \mathrm{D}$ and idealized, it allows us to have resolution fine enough to explicitly simulate small-scale internal waves, wave breaking, and turbulence (Fig. 4). A three-dimensional (3D) flow over 2D finite-amplitude topography has been shown to be quantitatively different from that over 1D topography, because 2D topography cannot only block the upstream flow, but also split it, further reducing the amplitude of the radiating internal lee waves (Nikurashin et al. 2014). In this study, we not only simulate the generation of internal waves as in Nikurashin et al. (2014), but also focus on their downstream evolution and dissipation and hence choose to run 2D simulations with high resolution (the resolution we cannot afford in $3 \mathrm{D}$ simulations) to explicitly simulate nonlinear wavewave interactions and wave breaking. Although the dissipation of internal lee waves in our model can be directly affected by viscosity (Shakespeare and Hogg 2017), the wave energy transfer to small dissipation scales, where viscous dissipation is most effective, is dominated by the nonlinear interactions. Figure 3 shows that changing the explicit numerical viscosity value by a factor of 2 to both high and low values leads to only about $10 \%-20 \%$ change in the total energy dissipation. This implies that, when viscosity is changed, the flow velocity gradients are changed correspondingly by the nonlinearity so that the resulting energy dissipation rate remains nearly unchanged.

To study the downstream evolution of internal waves and turbulence and investigate the mechanisms leading to their remote dissipation, we run three additional experiments. In the "rough and flat topography" experiment, we flatten out bottom topography in the region from 17 to $45 \mathrm{~km}$, while keeping rough topography upstream unchanged. This is our main perturbation experiment, which allows us to investigate the downstream evolution and remote dissipation of internal waves and turbulence generated at rough topography. This experiment configuration is idealized, but it allows us to separate the wave generation by the rough topography from the processes governing the downstream wave evolution and wave energy dissipation. Analysis of statistical properties of abyssal hills in the Southern Ocean shows that abyssal hill roughness can vary by up to a factor of 3-5 over the 50-100-km distance (Goff et al. 1997). In the "top sponge layer" experiment, in addition to the flat topography region, we replace the free-surface top boundary condition with a $5-\mathrm{km}$-thick sponge layer, in
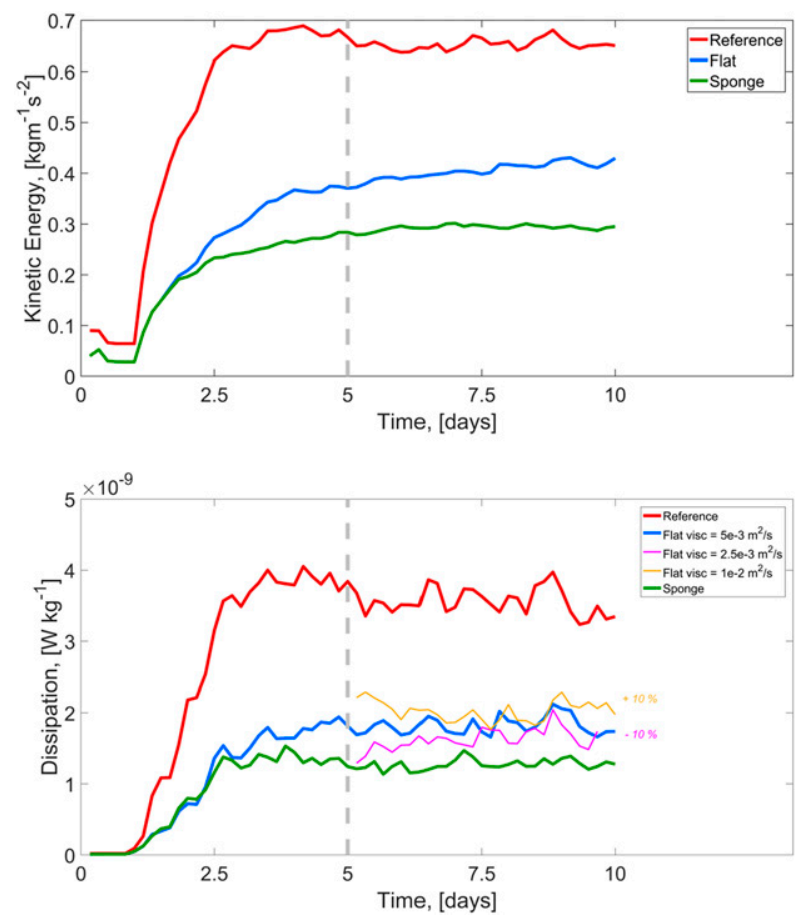

FIG. 3. The evolution of the volume-averaged (top) kinetic energy $\left(\mathrm{kg} \mathrm{m}^{-1} \mathrm{~s}^{-2}\right)$ and (bottom) energy dissipation rate ( $\mathrm{W} \mathrm{kg}^{-1}$ ) of perturbations showing the spinup and equilibration of all simulations.

which the flow perturbations are absorbed with a 1-h restoring time. This experiment allows us to study the role of reflection at the upper boundary and the contribution of the reflected waves to the downstream wave propagation and remote dissipation. Finally, in the "small-amplitude topography" experiment, we keep the flat topography region and top sponge layer, and reduce the amplitude of topography by a factor of 100 . This experiment allows are to simulate the evolution of linear lee waves.

\section{c. Flow decomposition and energy diagnostics}

To analyze the wave and turbulence response to the geostrophic flow over rough bottom topography, we decompose the model solution into the mean and perturbation components as,

$$
\mathbf{u}=\mathbf{U}_{0}+\mathbf{u}^{\prime}, \quad p=P_{0}+p^{\prime},
$$

where $\mathbf{u}=(u, v, \omega)$ and $p$ are the total velocity and pressure, $\mathbf{U}_{0}=\left(U_{0}, 0,0\right)$ and $P_{0}$ are the mean geostrophic flow and initial hydrostatic pressure, and $\mathbf{u}^{\prime}=\left(u^{\prime}, v^{\prime}, \omega^{\prime}\right)$ and $p^{\prime}$ are the velocity and pressure perturbations, respectively. The perturbation velocity $\mathbf{u}^{\prime}$ includes all motions generated by the interaction of the mean flow $\mathbf{U}_{0}$ with rough bottom topography, such as 


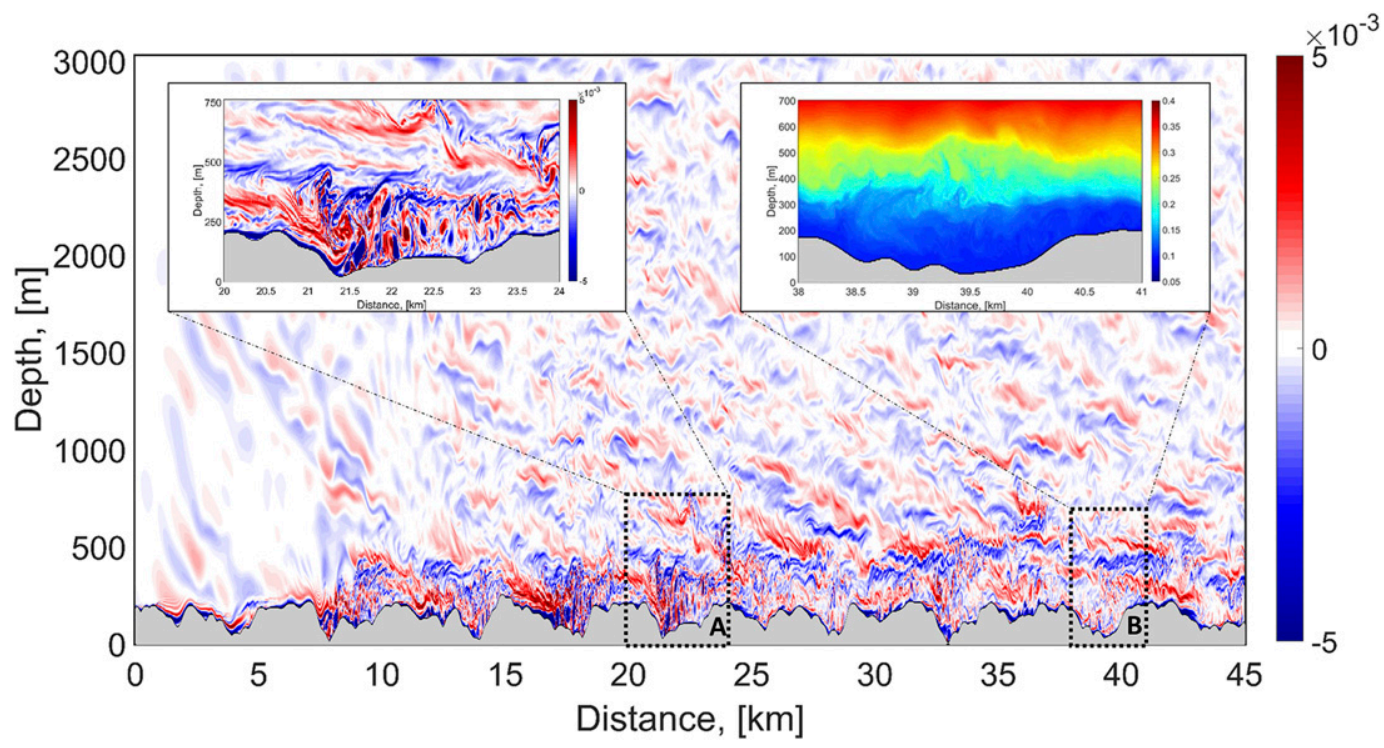

FIG. 4. A snapshot of vorticity $\left(\mathrm{s}^{-1}\right)$ from the reference simulation. The inset plot on the left is a zoom in on region A shown with the dashed black line. The inset on the right is temperature $\left({ }^{\circ} \mathrm{C}\right)$ from region $\mathrm{B}$. This snapshot illustrates small-scale internal waves, wave breaking, and turbulence explicitly simulated by the model.

steady and time-dependent internal waves, inertial oscillations, and turbulence.

In section 3, we use the energy budget diagnostics to characterize the intensity and distribution of internal waves and turbulence generated by geostrophic flow-topography interaction. The leading-order, timeaveraged energy equation for perturbations is

$$
U_{0} \frac{\partial \mathrm{TKE}}{\partial x}=-\frac{\partial \overline{u^{\prime} p^{\prime}}}{\partial x}-\frac{\partial \overline{\omega^{\prime} p^{\prime}}}{\partial z}+\rho_{0} \varepsilon
$$

where TKE is the turbulent kinetic energy, that is, kinetic energy of the perturbations, $\varepsilon$ is the dissipation of kinetic energy, and the overbar denotes the time average. The terms in this equation from left to right are the turbulent kinetic energy advection by the mean flow $U_{0}$, the horizontal and vertical energy radiation, and the energy dissipation $\varepsilon$. Energy transport by triple correlation terms and the energy conversion into potential energy have been shown to be small (Nikurashin and Ferrari 2010a) and hence are neglected. TKE is calculated as

$$
\mathrm{TKE}=\frac{1}{2} \rho_{0}\left(\overline{u^{\prime 2}}+\overline{v^{\prime 2}}+\overline{\omega^{\prime 2}}\right),
$$

where $\rho_{0}$ is a reference density. The energy dissipation $\varepsilon$ is calculated as

$$
\varepsilon=A_{i} \overline{\left(\frac{\partial u_{j}^{\prime}}{\partial x_{i}}\right)^{2}},
$$

where $A_{i}$ is horizontal and vertical viscosity.

\section{Results}

In this section, we first describe our reference experiment with a uniformly rough topography and show that the results are consistent with similar simulations used in previous studies and with observations in the Drake Passage region of the Southern Ocean. Then, we present a series of experiments with a flat topography region downstream of rough topography that we use to investigate the downstream evolution of internal waves. Finally, we present the energy budget diagnostics that we use to quantify the wave generation at rough topography, and their local and remote energy dissipation.

\section{a. Reference experiment: Uniformly rough topography}

Our reference experiment has a uniform realistic topography extending throughout the whole domain from 0 to $45 \mathrm{~km}$. A snapshot of the zonal velocity of perturbations at 10 days after the wave field is fully equilibrated is shown in Fig. 5a. In the interior away from topography, lee wave patterns are clearly visible with their phase lines tilting against the mean flow, consistent with a linear lee wave theory. Apart from the first $5 \mathrm{~km}$ of the domain, where the flow response to topography first appears and develops, the wave field along the domain is statistically uniform, but rather complex. It is a superposition of multichromatic, time-dependent, and steady waves produced both by bottom topography and through 

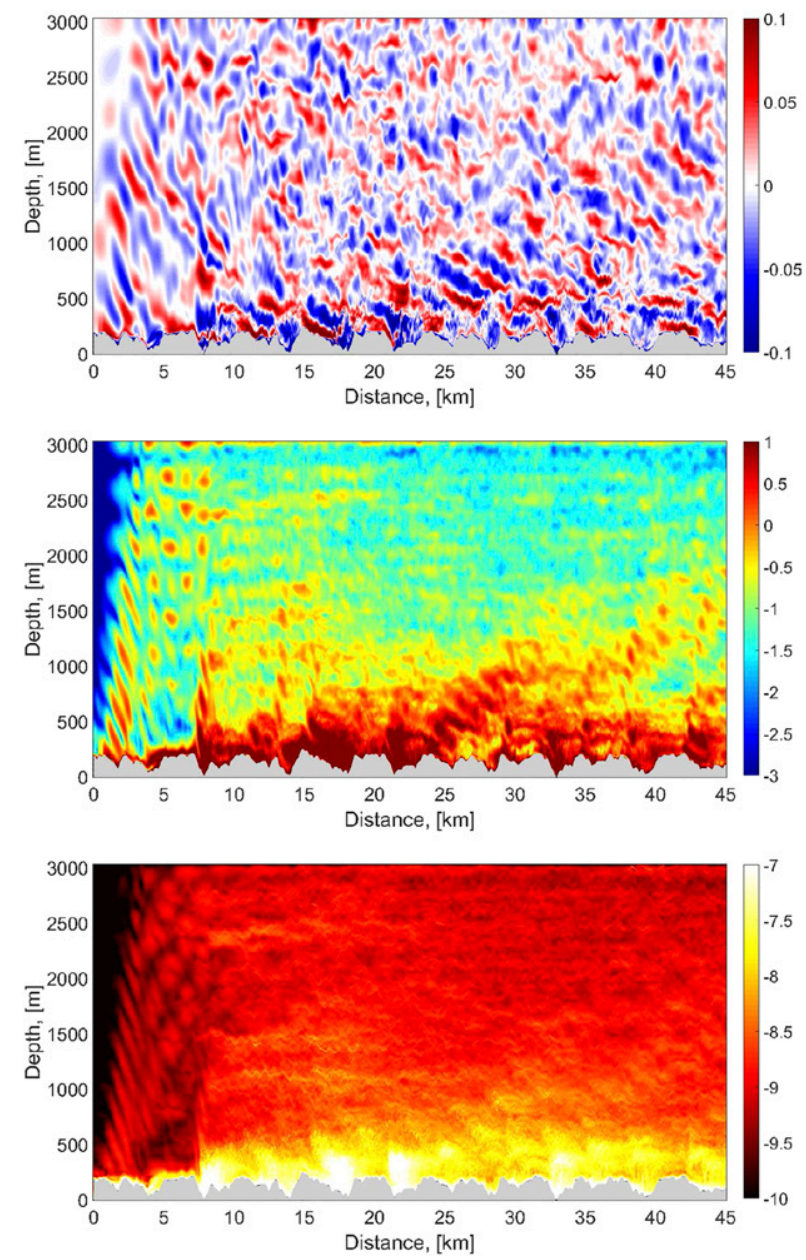

FIG. 5. The reference experiment: (a) a snapshot of the zonal velocity of perturbations $\left(\mathrm{m} \mathrm{s}^{-1}\right)$ at 10 days after the wave field is fully equilibrated; (b) time-mean TKE $\left[\log _{10}\left(\mathrm{~kg} \mathrm{~m}^{-1} \mathrm{~s}^{-2}\right)\right]$; and (c) time-mean dissipation $\varepsilon\left[\log _{10}\left(\mathrm{~W} \mathrm{~kg}^{-1}\right)\right]$.

various interactions in the interior. Waves reflecting from the upper boundary superimpose on the upward radiating waves, making the entire wave field even more irregular. In the bottom kilometer, the velocity field is particularly enhanced, indicating large-amplitude lee waves, which break and dissipate some of their energy right after their generation. Patches of very high velocity seen behind steep topographic features are associated with low-level, nonradiating, nonlinear motions such as topographically blocked flows (e.g., Trossman et al. 2015; Dossmann et al. 2016; Klymak 2018) and hydraulic jumps (e.g., Baines 1995). Overall, the results are consistent with previous numerical simulations (e.g., Nikurashin and Ferrari 2010b; Nikurashin et al. 2014) and show that the flow response is a superposition of different motions arising from the interaction of a uniform mean flow with realistic, multiscale rough topography.
To characterize the time-averaged flow response to rough topography, we also show the distribution of the time-averaged kinetic energy of perturbations, TKE, and their energy dissipation $\varepsilon$ (Figs. 5b,c). As with the zonal velocity snapshot in Fig. 5a, very high values of TKE, up to $10 \mathrm{~kg} \mathrm{~m}^{-1} \mathrm{~s}^{-2}$ (corresponding to up to $10^{-1} \mathrm{~m} \mathrm{~s}^{-1}$ speed), near the bottom indicate low-level, large-amplitude lee waves and strong nonlinear motions generated behind steep topographic features. TKE in the interior away from topography is generally lower by one to two orders of magnitude and is fairly uniform both in the horizontal and vertical. Unlike TKE, $\varepsilon$ depends not only on amplitudes of the perturbations, but also on their gradients, and hence is dominated by small-scale internal waves and turbulent motions, which leads to a more uniform timeaveraged horizontal distribution of $\varepsilon$ throughout the domain. $\varepsilon$ is enhanced up to $10^{-7} \mathrm{~W} \mathrm{~kg}^{-1}$ above topography, where the flow perturbations are generated. This enhancement is again associated with the breaking of low-level lee waves and hydraulic flows. Consistent with observations, $\varepsilon$ is one to two orders of magnitude smaller in the interior, varying from $10^{-9}$ to $10^{-8} \mathrm{~W} \mathrm{~kg}^{-1}$, which is still an order of magnitude larger than the background ocean energy dissipation of $10^{-10} \mathrm{~W} \mathrm{~kg}^{-1}$ (e.g., Naveira Garabato et al. 2004).

To quantify the horizontal distribution of internal waves and turbulence we look at the variation of the TKE and $\varepsilon$ as a function of distance for the bottom region and the interior of the domain (Fig. 8). We define the bottom region as the bottom $1000 \mathrm{~m}$ and the interior as the region $1000-3000 \mathrm{~m}$ above bottom and integrate TKE and $\varepsilon$ over these two regions. TKE in the bottom region is statistically uniform along the domain, but can still vary by up to an order of magnitude, depending on the amplitude of underlying topography: high values of TKE are associated with energetic lee waves and strong low-level nonlinear motions close to the topographic features that generate them. In the interior, the overall TKE level is lower and more uniform along the domain, as this region is away from topography and the wave field is much less coherent than near topography (Fig. 5a). The distribution of $\varepsilon$ along the domain is generally similar to that of the TKE, because it is dominated by small-scale internal waves and turbulence, which result from the instabilities and downscale energy cascade from larger scale internal waves, motions that dominate TKE.

Finally, to quantify the vertical distribution of $\varepsilon$ we show the horizontally averaged, vertical profiles of energy dissipation in Fig. 9. The averaged value of the energy dissipation in the deep ocean reaches up to $10^{-7} \mathrm{~W} \mathrm{~kg}^{-1}$ near topography, a value consistent with 
pervious 2D numerical simulations and generally consistent with observations of turbulent energy dissipation in Drake Passage (Naveira Garabato et al. 2004). The energy dissipation decreases by about one to two orders of magnitude to $10^{-9} \mathrm{~W} \mathrm{~kg}^{-1}$ in the ocean interior, but it remains enhanced over the background level of energy dissipation of $10^{-10} \mathrm{~W} \mathrm{~kg}^{-1}$ in the Southern Ocean.

In summary, the results from the reference Drake Passage simulation show that internal waves and energy dissipation are generated in regions with strong bottom flow and rough topography. Internal wave amplitudes and energy dissipation values are generally consistent with observations. Both TKE and $\varepsilon$ are statistically uniform along the domain, which is expected, given that the bottom topography is uniformly rough along the domain.

\section{b. Rough and flat topography}

To investigate the downstream evolution and remote dissipation of internal waves and turbulence, we now analyze the rough and flat topography experiment designed to have a flat topography region following the rough topography region. A snapshot of the zonal velocity and time-mean TKE and $\varepsilon$ from this experiment are shown in Fig. 6. Comparing this simulation to our reference experiment with uniformly rough topography, we can see that the wave field over the rough topography region, $7-17 \mathrm{~km}$, is similar between the two experiments. Small differences in this region are likely caused by the upstream influence from the waves which are generated in the region from 17 to $45 \mathrm{~km}$ in the reference experiment, but not in the rough and flat topography experiment. Despite the absence of the topographic wave generation in the flat topography region, strong multichromatic wave field with amplitudes up to $5-10 \mathrm{~cm} \mathrm{~s}^{-1}$ can be clearly seen in the interior of this region. The evolution of the wave field and the propagation of internal waves into the flat topography region are illustrated by a sequence of snapshots of zonal velocity shown in Fig. 7. We can see that the downstream propagation of the internal wave field is a combination of the upward radiation of waves and their downstream advection by the mean flow.

The time-mean TKE and $\varepsilon$ are also similar over the rough topography region between the two experiments. Over the flat topography region, TKE and $\varepsilon$ decrease with the distance away from the rough topography region, but they remain enhanced above the background level for tens of kilometers downstream: $\varepsilon$ varies from $10^{-9}$ to $10^{-8} \mathrm{~W} \mathrm{~kg}^{-1}$ in the interior over flat topography, which is one to two orders of magnitude above the ocean background energy dissipation level. To maintain internal waves and turbulent energy dissipation over the flat topography region the wave energy must be carried
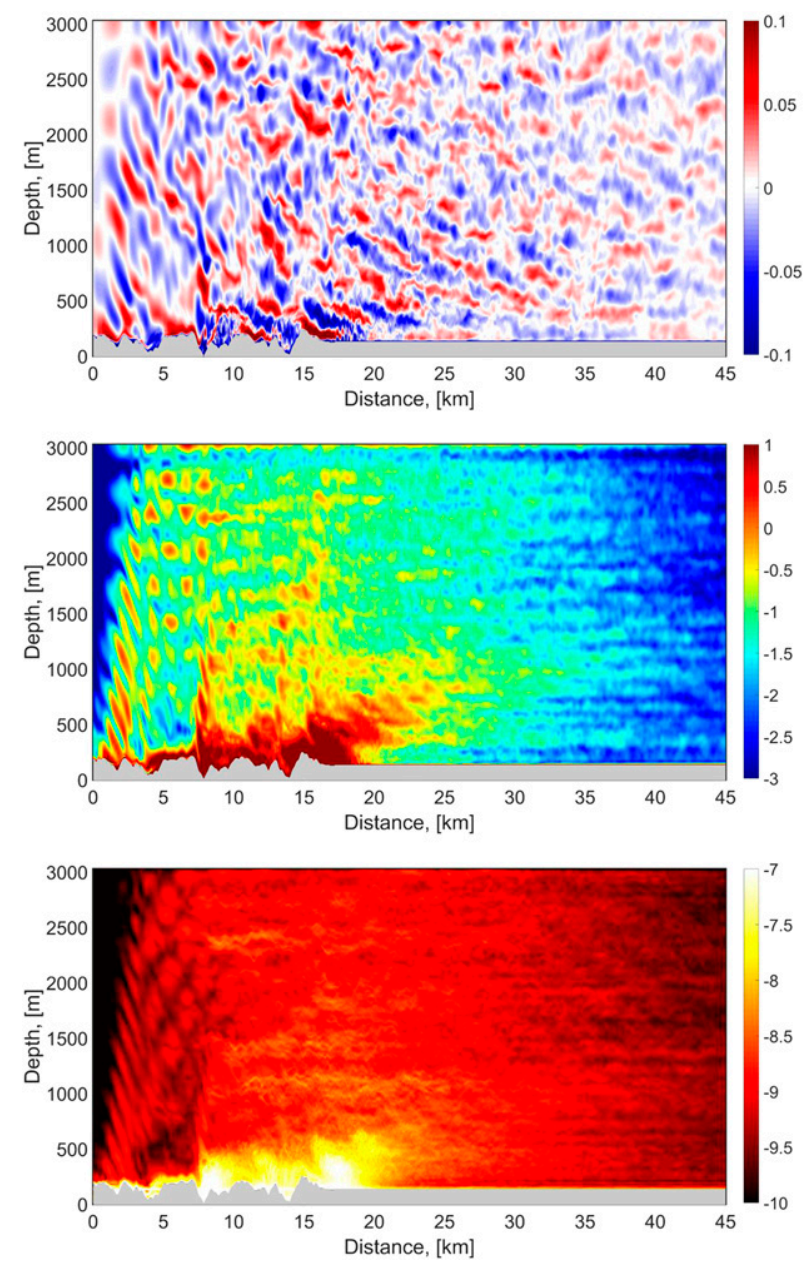

FIG. 6. The rough and flat topography experiment: (a) a snapshot of the zonal velocity of perturbations $\left(\mathrm{m} \mathrm{s}^{-1}\right)$ at 10 days after the wave field is fully equilibrated; (b) time-mean TKE $\left[\log _{10}\left(\mathrm{~kg} \mathrm{~m}^{-1} \mathrm{~s}^{-2}\right)\right.$; and (c) time-mean dissipation $\varepsilon\left[\log _{10}\left(\mathrm{~W} \mathrm{~kg}^{-1}\right)\right]$.

downstream into this region by the wave radiation and the mean flow advection.

The horizontal decay of TKE and $\varepsilon$ is quantified in Fig. 8. Over the flat topography region, the TKE gradually decrease with distance away from the generation site. From the slope of the decay curve, we estimate that an $e$-folding decay length scale is about $3 \mathrm{~km}$ for the bottom region and up to $20-30 \mathrm{~km}$ for the interior. The decay length scale is shorter for the bottom region, because the flow in this region is dominated by nonradiating, small-scale turbulent motions which have a short residence time scale after they are generated and hence are not propagated far downstream before they completely dissipate. After about $10 \mathrm{~km}$ downstream of rough topography, the decay scale in the bottom layer becomes similar to that in the interior. In the interior region dominated 

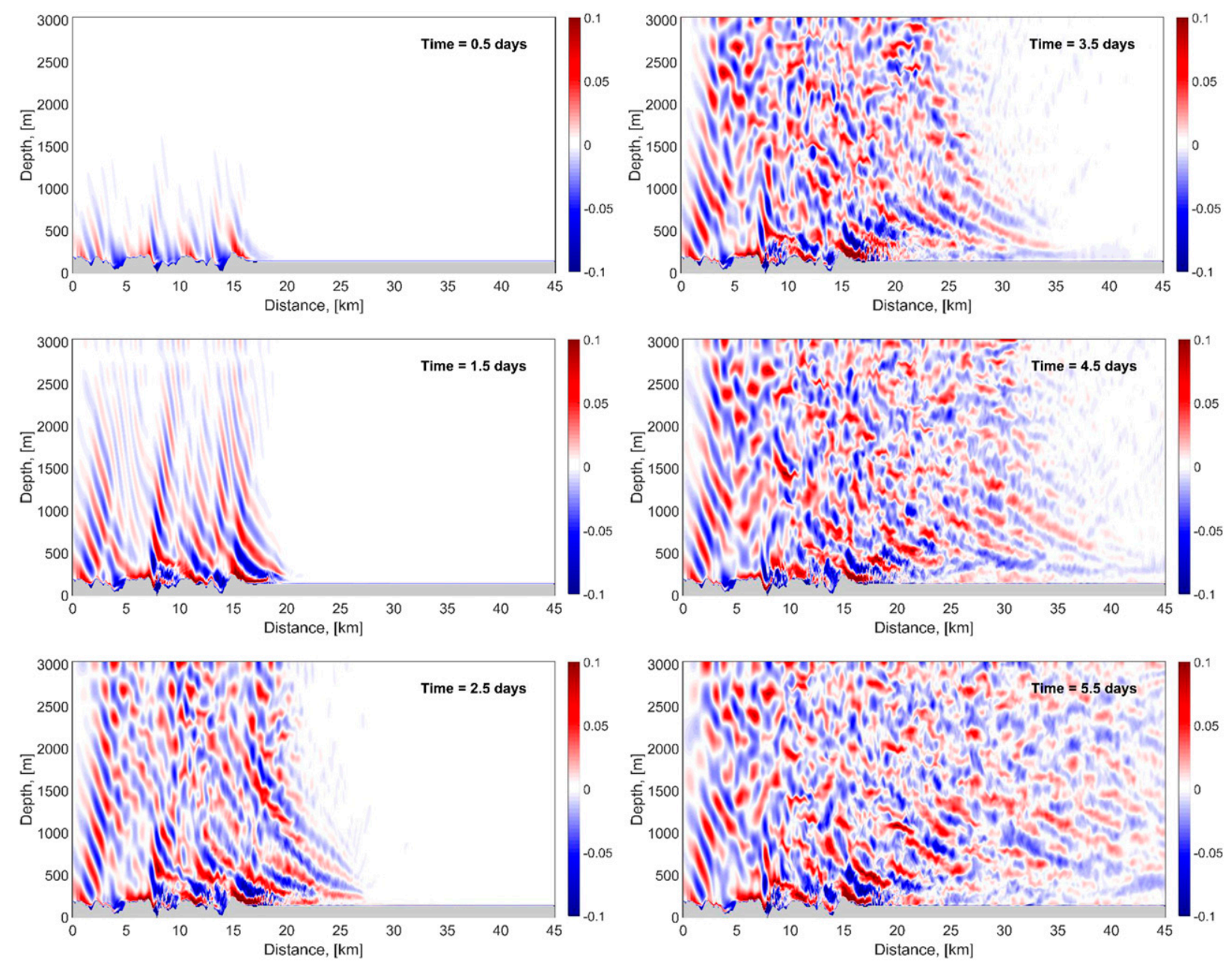

FIG. 7. Evolution of the zonal velocity of perturbations $\left(\mathrm{m} \mathrm{s}^{-1}\right)$ from the rough and flat topography experiment.

by the internal wave response, the wave energy is propagated much further downstream before it is dissipated, therefore powering turbulence and mixing tens of kilometers away from the rough topography region.

Vertical profiles of the horizontally averaged energy dissipation rates for three regions (rough topography, near-field and far-field downstream of rough topography) along the domain are shown in Fig. 9. Over the rough topography region, $7-17 \mathrm{~km}$, similar dynamical regimes take place in these two experiments so that the vertical profiles of dissipation are very close. In the near-field region over flat topography, $17-27 \mathrm{~km}$, the overall vertical profile appears as energy dissipation decaying away from the bottom with an order of magnitude enhancement in the bottom kilometer, similar to that in the rough topography region, but this energy is propagated from the region upstream rather than generated locally. While the bottom rate of the energy dissipation is reduced over the flat topography region, the interior energy dissipation rates are comparable between the flat and rough topography regions. This profile of the energy dissipation is sustained by the mean flow advecting both strong turbulence near the bottom and internal waves in the interior downstream from the rough topography region. In the far-field region over flat topography, $35-45 \mathrm{~km}$, the vertical profile of energy dissipation becomes fairly uniform in the vertical. The nonradiating, low-level turbulent motions decay rapidly away from the rough topography region and the response in this region is dominated by internal waves, which become quite uniformly distributed in the vertical at this distance.

In summary, our results show that significant amount of kinetic energy and energy dissipation occurs over flat topography tens of kilometers downstream of the rough topography region. The energy dissipation over flat topography appears as decaying 

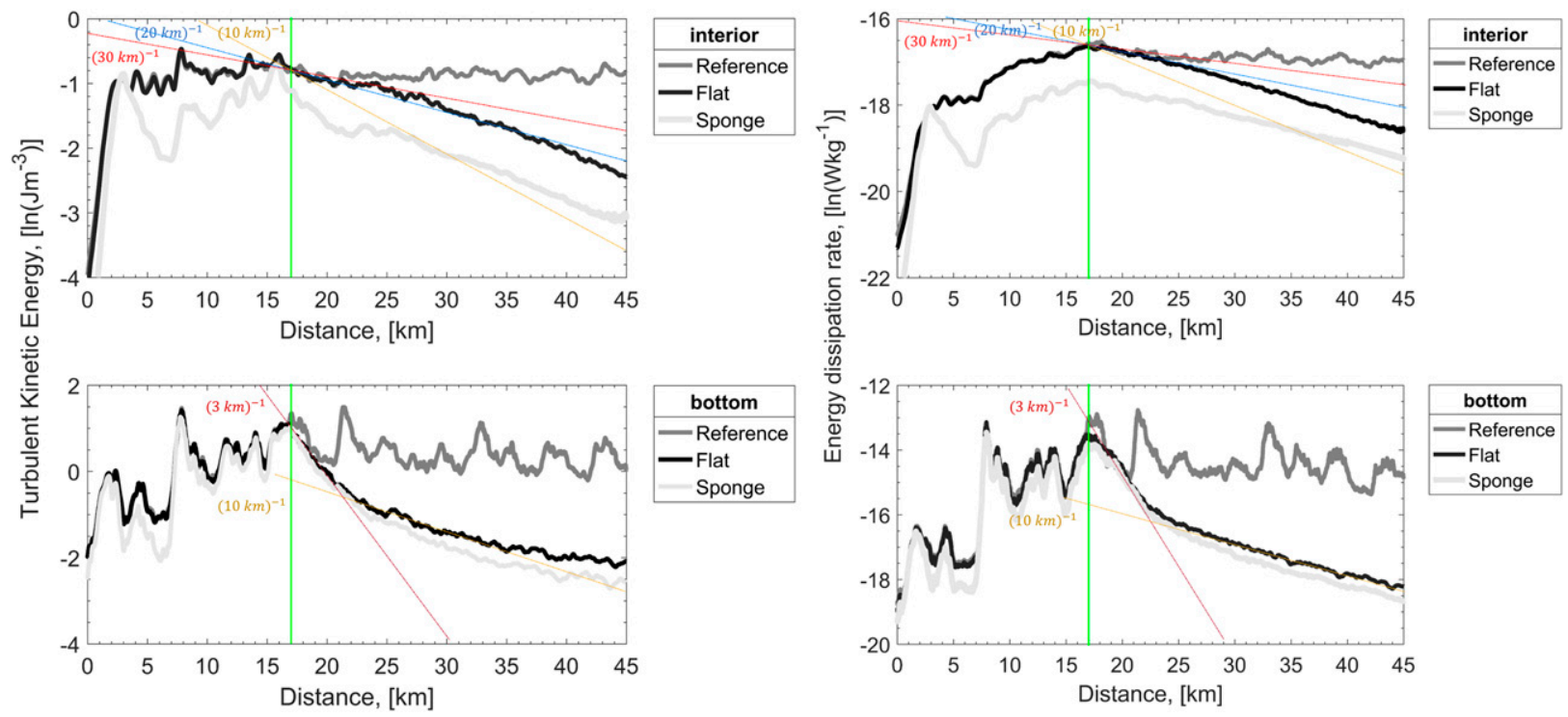

FIG. 8. The horizontal distribution of (left) TKE $\left[\ln \left(\mathrm{kg} \mathrm{m}^{-1} \mathrm{~s}^{-2}\right)\right]$ and (right) $\varepsilon\left[\ln \left(\mathrm{W} \mathrm{kg}^{-1}\right)\right]$ averaged over the (top) interior and (bottom) bottom regions. We define the bottom region as the bottom $1000 \mathrm{~m}$ and the interior as the region from 1000 to $3000 \mathrm{~m}$ above bottom.

away from the bottom from $10^{-8} \mathrm{~W} \mathrm{~kg}^{-1}$ near the bottom to $10^{-9} \mathrm{~W} \mathrm{~kg}^{-1}$ in the interior, values consistent with observations. However, this vertical distribution of the energy dissipation is maintained by the downstream advection of internal waves and turbulence from the rough topography region, rather than by the local wave generation and vertical radiation, as in the rough topography region.

\section{c. Top sponge layer}

The rough and flat topography experiment shows that significant levels of internal wave and turbulent energy are present over the flat topography region. This energy is propagated downstream from the rough topography region, where internal waves are generated. Internal waves are advected into the flat topography region as they propagate upward throughout the interior (Fig. 7). When the waves reach the top boundary, they are reflected and propagate back down, further contributing to the internal wave kinetic energy and energy dissipation downstream of the rough topography region. To investigate the role of the wave reflection at the upper boundary for the downstream wave propagation and remote dissipation, we analyze the experiment with a sponge layer at the upper boundary.

A snapshot of the wave zonal velocity, time-averaged TKE and $\varepsilon$ are shown in Fig. 10. We can see that the wave field in the ocean interior becomes much more regular in this case as the waves are absorbed in the sponge layer when they reach the upper boundary and only the upward propagating waves (with phase lines tilting against the mean flow) are present. As a result, there is a decrease of both TKE and $\varepsilon$ over the flat topography region compared to the rough and flat topography experiment.

The horizontal distribution of TKE in the boundary and interior regions shows that there is still some energy left over the flat topography region, but the TKE and $\varepsilon$ levels are about a factor of 2 lower than those in the rough and flat topography experiment. The effect of reflection at the upper boundary is stronger in the interior than in the bottom region, because it is dominated by internal waves.

Vertical profiles of $\varepsilon$ from this experiment are shown in Fig. 11. We can see that while the energy dissipation still decays away from the bottom into the interior of the domain over the flat topography region and the rate of the energy dissipation near the bottom is nearly the same, the rate of the energy dissipation in the interior is reduced by about a factor of 2 . This experiment indicates that the wave reflection at the upper boundary is a major contributor to the downstream wave energy propagation and energy dissipation: reflection at the upper boundary allows all wave energy to stay in the domain and hence it enhances TKE and $\varepsilon$ downstream of the rough topography region.

\section{d. Small-amplitude topography}

Linear theory predicts that lee waves propagate upward away from topography and have their constant phase lines tilting against the mean flow, implying that linear lee waves should not be present over the flat topography region. To verify that the downstream 


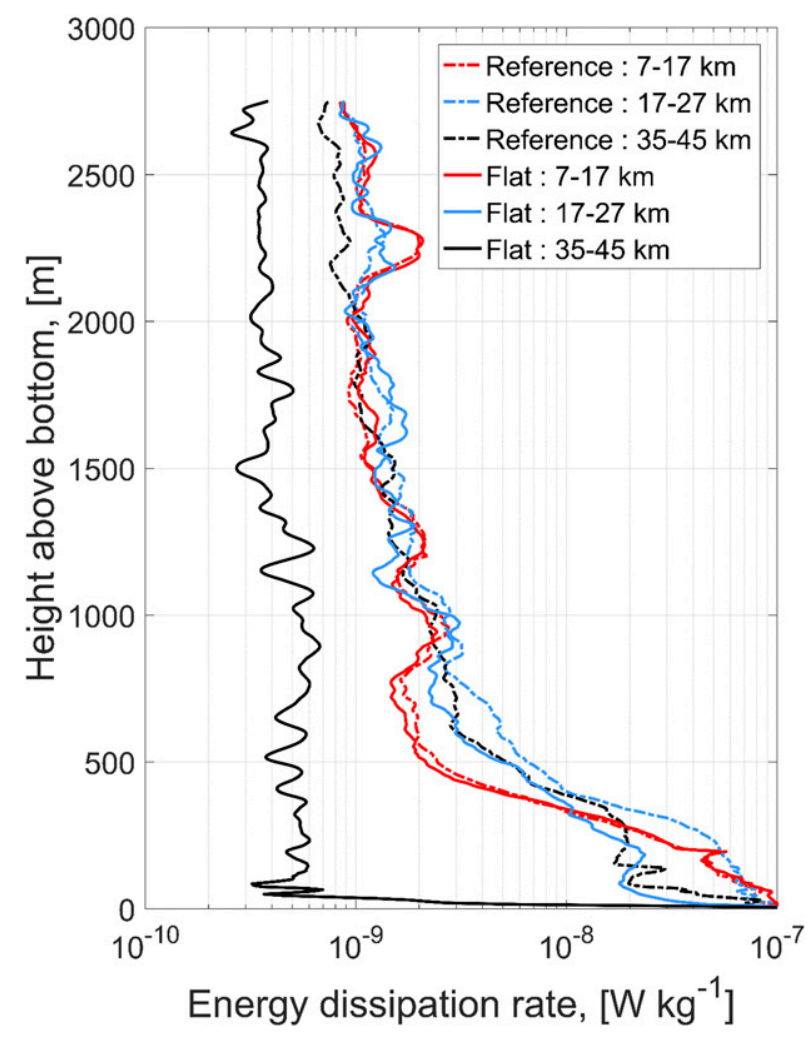

FIG. 9. Vertical profiles of the horizontally averaged energy dissipation rates $\left(\mathrm{W} \mathrm{kg}^{-1}\right)$ for three regions of the domain from the reference (dashed) and the rough and flat topography experiments (solid).

propagation of internal waves and their remote dissipation is a result of a nonlinear flow response leading to internal waves that are not locked against the mean flow and can get advected downstream, we analyze the small-amplitude topography experiment.

A snapshot of zonal velocity and time-averaged TKE are shown in Fig. 12. Over the rough topography region, linear lee wave patterns are clearly visible with the dominant horizontal and vertical scales of $2 \mathrm{~km}$ and $500 \mathrm{~m}$, respectively, and constant phase lines tilting upstream, consistent with a linear theory (e.g., Nikurashin and Ferrari 2011; Scott et al. 2011). The amplitudes of the waves do not decay significantly with height, consistent with no significant energy dissipation in this small-amplitude, linear wave limit. Over the flat topography region, internal wave patterns are still visible near the bottom, likely because of a weakly nonlinear generation still taking place, but their amplitudes are an order of magnitude smaller compared to wave amplitudes over rough topography region. This experiment indicates that lee waves generated over rough topography do not radiate downstream and propagate their energy into the flat topography region.
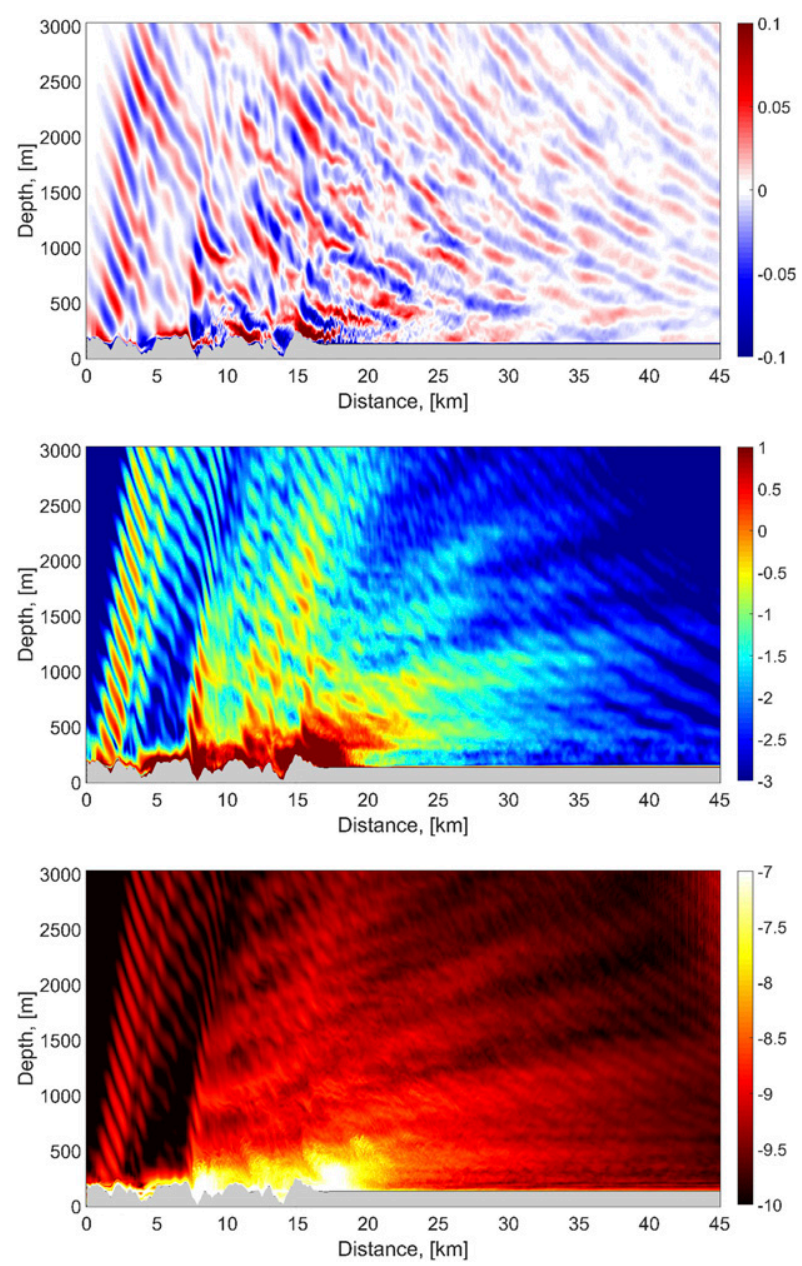

FIG. 10. The top sponge layer experiment: (a) a snapshot of the zonal velocity of perturbations $\left(\mathrm{m} \mathrm{s}^{-1}\right)$ at 10 days after the wave field is fully equilibrated; (b) time-mean TKE $\left[\log _{10}\left(\mathrm{~kg} \mathrm{~m}^{-1} \mathrm{~s}^{-2}\right)\right]$; and (c) time-mean dissipation $\varepsilon\left[\log _{10}\left(\mathrm{~W} \mathrm{~kg}^{-1}\right)\right]$.

This result is consistent with the linear lee wave theory prediction and suggests that the energy dissipation taking place downstream of rough topography region results from the mean flow advection of internal waves and turbulence produced by nonlinear wave generation and wave-wave interactions. While linear lee waves are stationary with respect to the flow that generates them and hence cannot be advected downstream by the mean flow, waves and turbulence resulting from various nonlinear interactions do not have to be stationary and therefore can be carried downstream.

\section{e. Energy budget}

Our results show that internal waves and turbulence generated over the rough topography region can propagate tens of kilometers downstream and dissipate remotely over the flat topography region. To quantify the local and remote energy dissipation, 


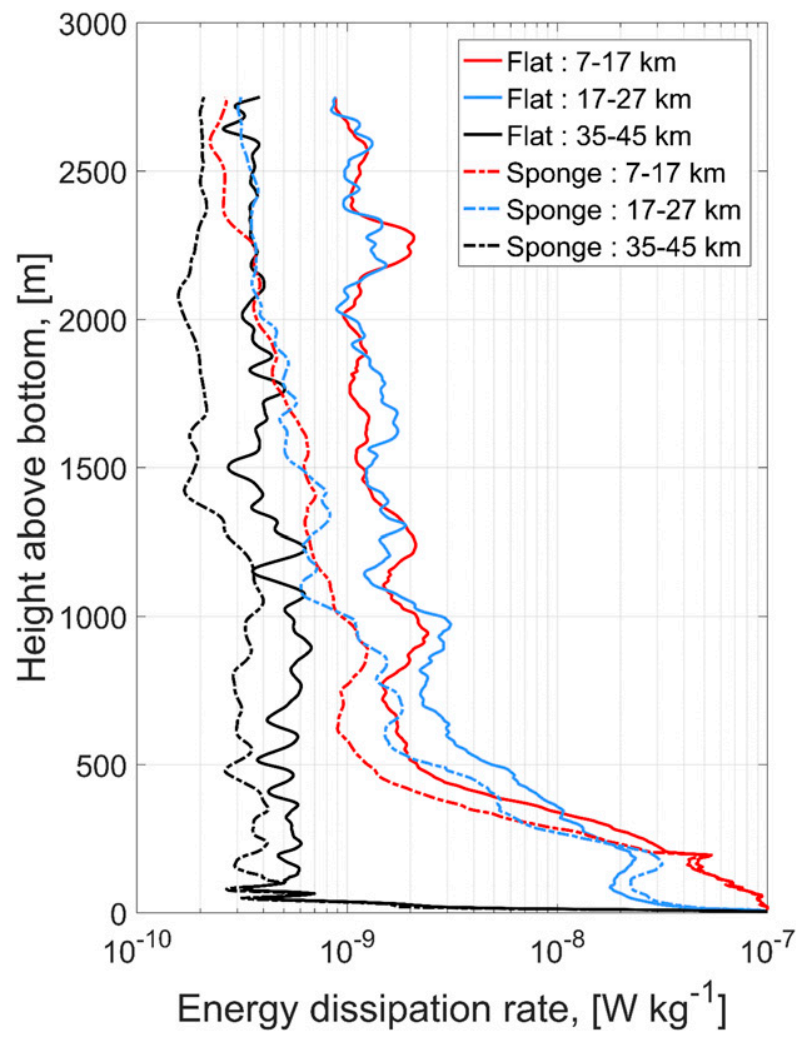

FIG. 11. Vertical profiles of the horizontally averaged energy dissipation rates in $\left(\mathrm{W} \mathrm{kg}^{-1}\right)$ for three regions of the domain from the rough and flat topography (solid) and the top sponge layer experiments (dashed).

we estimate the TKE budget for the rough and flat topography regions (Fig. 13). The TKE budget is obtained by integrating (3) over a closed volume that spans in the vertical from the level of topography (300-m height) to either the ocean surface or the bottom of the sponge layer (3-km height). Since the budget is calculated after the wave field is fully equilibrated, changes of the integrated TKE in time are small. The total budget is closed within $5 \%-10 \%$ of the bottom energy flux value (i.e., the energy source term) with the residual representing an error bar, which accounts for errors in the budget calculation, unsteadiness in the wave field, and possible spurious numerical dissipation.

For the volume over rough topography, $5.8 \mathrm{~mW} \mathrm{~m}^{-2}$ of energy is generated near topography and radiated vertically into the interior as the mean flow interacts with the bottom topography. $2.1 \mathrm{~mW} \mathrm{~m}^{-2}$ (or $36 \%$ of the generated energy) dissipates within the volume above the rough topography region. Energy fluxes on the upstream boundary of the volume consist of $0.9 \mathrm{~mW} \mathrm{~m}^{-2}$ radiating upstream against the mean flow and $0.6 \mathrm{~mW} \mathrm{~m}^{-2}$ being advected downstream by the mean flow. Therefore, $0.3 \mathrm{~mW} \mathrm{~m}^{-2}$ is
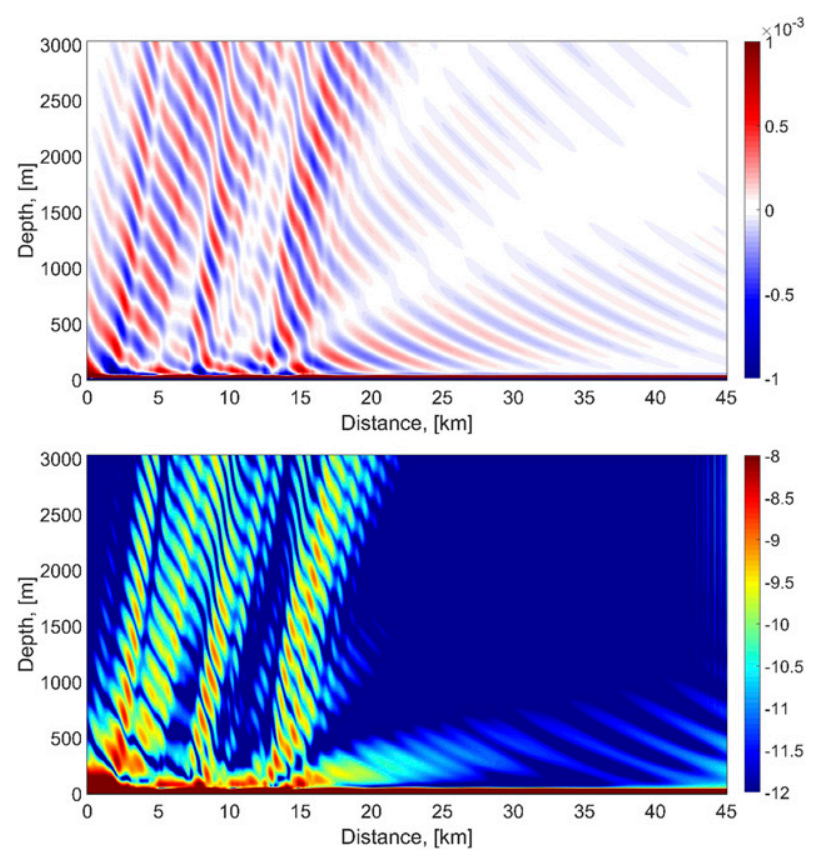

FIG. 12. The small-amplitude topography experiment: (a) a snapshot of the zonal velocity of perturbations $\left(\mathrm{m} \mathrm{s}^{-1}\right)$ at 10 days after the wave field is fully equilibrated; (b) time-mean TKE $\left[\log _{10}\left(\mathrm{~kg} \mathrm{~m}^{-1} \mathrm{~s}^{-2}\right)\right]$.

being propagated from the rough topography region into the sponge layer upstream. This number is small as most of the lee wave energy is expected to radiate upward and get advected downstream by the mean flow. Energy fluxes on the downstream boundary of this volume, on the other hand, consist of $4.7 \mathrm{~mW} \mathrm{~m}^{-2}$ of downstream energy advection by the mean flow and $1.6 \mathrm{~m} \mathrm{~W} \mathrm{~m}^{-2}$ of upstream energy radiation. Advection by the mean flow dominates, resulting in $3.1 \mathrm{~mW} \mathrm{~m}^{-2}$ (or $53 \%$ of the generated energy) propagating downstream into the flat topography region. For the volume over flat topography, the vertical energy flux at the bottom is nearly zero, $0.3 \mathrm{~mW} \mathrm{~m}^{-2}$, consistent with flat topography and no wave generation. The total energy dissipation in this volume is $3.1 \mathrm{~mW} \mathrm{~m}^{-2}$. Energy fluxes on the downstream boundary of the volume consist of $0.5 \mathrm{~mW} \mathrm{~m}^{-2}$ of energy advected into the sponge layer and $0.1 \mathrm{~mW} \mathrm{~m}^{-2}$ radiating back into the volume. Hence, the energy budget indicates that for the TKE generated at the rough bottom topography, $53 \%$ of the generated energy propagates downstream and dissipates remotely and only $36 \%$ dissipates locally over the rough topography, wave generation site.

To quantify the role of the wave reflection at the upper boundary for the wave propagation and energy dissipation, the TKE budgets for the two regions from the rough and flat topography experiment with a top 


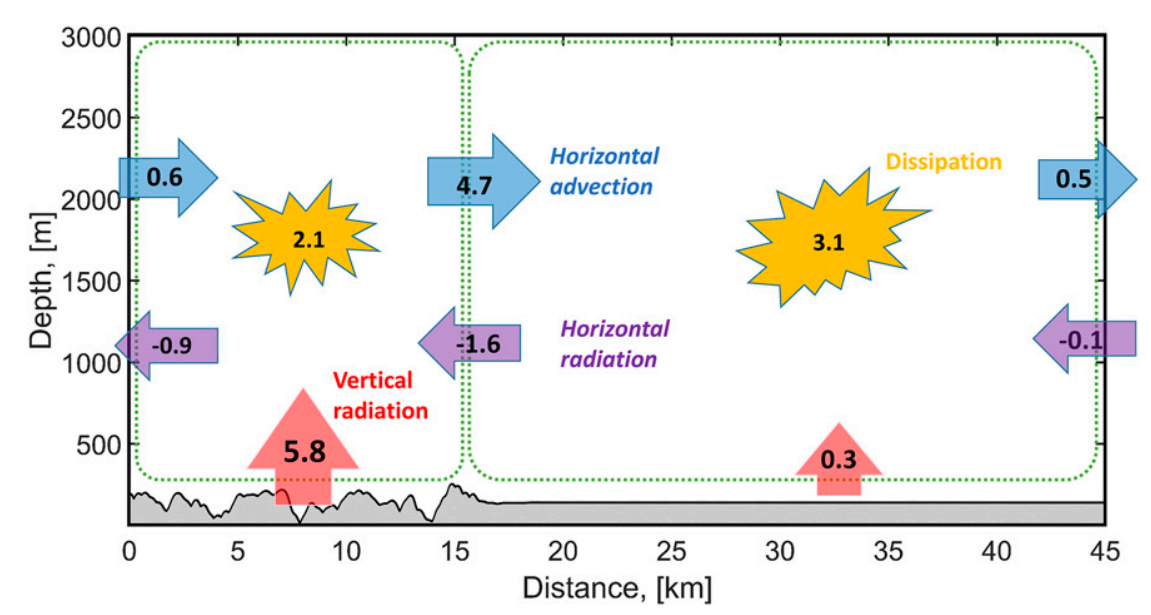

FIG. 13. TKE budget $\left(\mathrm{mW} \mathrm{m}^{-2}\right)$ for the rough and flat topography experiment.

sponge layer are presented in Fig. 14. The magnitude of the vertical energy flux is about the same, $5.7 \mathrm{~mW} \mathrm{~m}^{-2}$, for the same topography and mean flow. A slight difference can be explained by the fact that there are no reflected waves that can radiate back to topography and modify the wave generation. The values of dissipation rates are $1 \mathrm{~mW} \mathrm{~m}^{-2}$ and $1.9 \mathrm{~mW} \mathrm{~m}^{-2}$ in the rough and flat topography regions, respectively. They are significantly decreased in both regions as part of the TKE is absorbed in the sponge layer, when the waves reach the upper boundary. A fraction of the local energy dissipation decreases to $18 \%$, indicating that reflected internal waves play a significant role for the local energy dissipation. A total of $2.4 \mathrm{~mW} \mathrm{~m}^{-2}$ (or $42 \%$ of the generated energy) is propagated downstream into the flat topography region, where most of this energy dissipates.

In summary, these results indicate that the horizontal advection of the mean flow is the main contributor to the nonlocal dissipation of TKE generated by geostrophic flows impinging on rough bottom topography. The wave reflection at the upper boundary plays a smaller, but an important role in enhancing this energy propagation.

\section{Conclusions}

Recent observations show enhanced internal wave kinetic energy and turbulent energy dissipation in the bottom kilometer in the frontal regions of the ACC. Idealized numerical simulations and linear theory assume spatially uniform and periodic topography and tend to overestimate the observed levels of the energy dissipation locally at the generation sites. In this study, we use a series of numerical, realistic-topography simulations and parameters typical of Drake Passage to test this assumption and explore the downstream propagation and remote energy dissipation of internal waves.

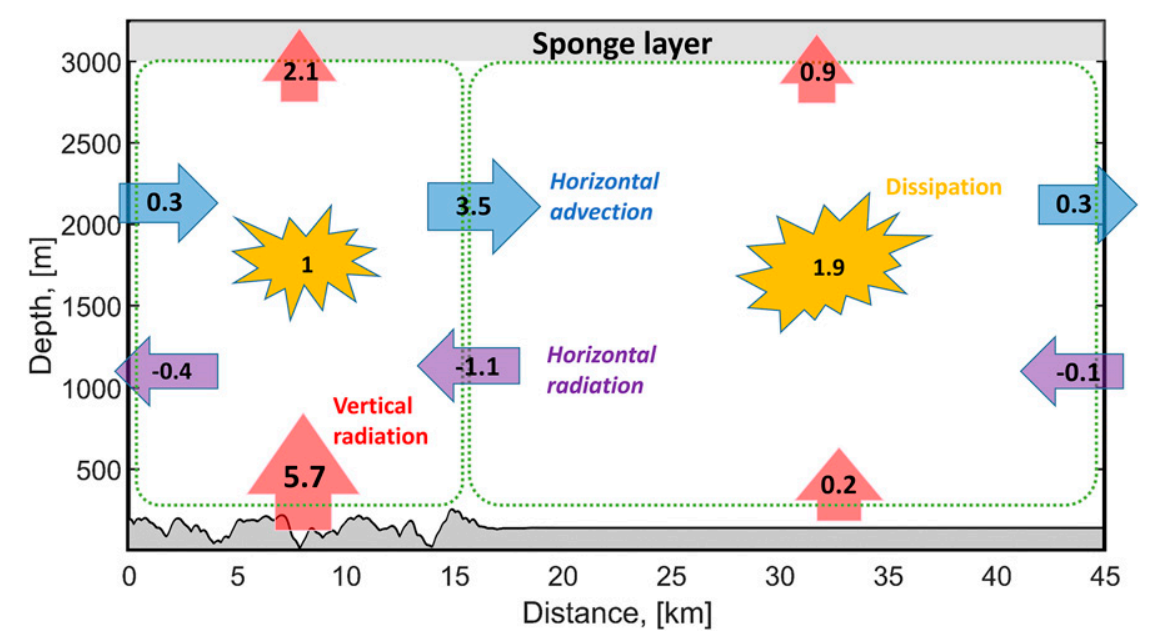

FIG. 14. TKE budget $\left(\mathrm{mW} \mathrm{m}^{-2}\right)$ for the top sponge layer experiment. 
Our results show that, when a mean flow impinges on rough bottom topography, internal waves not only radiate upward and dissipate their energy locally above topography, but also get advected downstream of the rough topography region and dissipate their energy remotely. The mean flow advects the wave field downstream, while the wave-wave interactions move energy to smaller scales. So, the two competing processes, the advection by mean flow and nonlinear energy transfer to small scales, determine the horizontal decay length scale of wave kinetic energy and energy dissipation downstream of the rough topography wave generation site. We find that only $30 \%-40 \%$ of the energy dissipation occurs locally over the rough topography region. Roughly one-half of this energy dissipation can be attributed to the upward radiating internal waves and the other half to the waves reflected back into the interior at the upper boundary. Over $50 \%$ of the wave energy generated at rough topography is advected downstream of the rough topography region, where this energy eventually dissipates. The internal wave kinetic energy and energy dissipation decay away from the generation site with an $e$-folding length scale of $20-30 \mathrm{~km}$. The vertical energy dissipation profile in the region downstream of the generation site appears as decaying away from the bottom, but it is sustained by the horizontal advection of internal waves and turbulence, rather than by the vertical wave radiation as in the rough topography region. Wave reflection at the upper boundary also contributes to the energy dissipation downstream of the wave generation site, enhancing the energy dissipation rates in the interior by up to a factor of 2 .

Our work suggests that a local balance between the wave generation at the bottom topography and turbulent energy dissipation in the interior aloft, often assumed in previous studies (e.g., Nikurashin and Ferrari 2010a,b; Waterman et al. 2013; Sheen et al. 2013; Melet et al. 2014, 2015), does not apply to the lee wave problem. As a result, when interpreting observations of turbulent energy dissipation, the topographic roughness tens of kilometers upstream of the measurement site needs to be taken into account. Observations can show less of the energy dissipation than predicted from the underlying topography, if the topography upstream is smoother, and vice versa. Combined with other proposed mechanisms, the advection of internal waves by mean flows and their remote dissipation can contribute to the reported difference between the predicted wave generation and the observed energy dissipation. From the global modeling perspective, being advected tens of kilometers downstream, the lee-wave-driven energy dissipation and mixing remain local in coarse-resolution, $O(100) \mathrm{km}$, global models, but become remote in high-resolution, $O(10) \mathrm{km}$, global ocean models.
There are several limitations of this work, which mainly result from the choice of the idealized model configuration we made to isolate the process. First, our simulations are $2 \mathrm{D}$ and the internal wave generation and dissipation processes are quantitatively different in $3 \mathrm{D}$ flows. Topography can be anisotropic in 3D, which can also modify the wave generation and dissipation processes quantitatively. In addition, 3D flows over topography can lead to qualitatively different motions such as low-level jets, flow separation and vortex shedding, processes which are absent in our 2D simulations, but could provide additional routes to small-scale dissipation near topography. Mean flows can also have shear in the deep ocean and hence can affect the wave radiation and dissipation, and hence can potentially influence the decay length scale of the wave kinetic energy and energy dissipation away from the wave generation site. A major limitation of our model is the use of the Laplacian horizontal and vertical friction with enhanced viscosity coefficients to represent the subgrid scale physics, which can potentially lead to the underestimation of the remote internal wave dissipation. These limitations are expected to result in quantitative changes of the results and can be addressed in future simulations.

Acknowledgments. We thank two anonymous reviewers and the editor for their useful comments and suggestions that improved the manuscript. KZ was supported by the China Scholarship Council (CSC). MN was supported by the Australian Research Council (ARC) Discovery Early Career Research Award (DECRA) Fellowship (DE150100937). This research was undertaken with the assistance of resources provided at the NCI National Facility systems at the Australian National University through the National Computational Merit Allocation Scheme supported by the Australian Government. We also thank Prof. C. Guan and J. Sun and the support from the National Key Research and Development Program of China (2016YFC1401405) and the National Natural Science Foundation of China (41276010, 41376010, and U1406401).

\section{REFERENCES}

Baines, P. G., 1995: Topographic Effects in Stratified Flows. Cambridge University Press, $500 \mathrm{pp}$.

Bell, T. H., 1975a: Lee waves in stratified flows with simple harmonic time dependence. J. Fluid Mech., 67, 705-722, https://doi.org/10.1017/S0022112075000560.

—_, 1975b: Topographically generated internal waves in the open ocean. J. Geophys. Res., 80, 320-327, https://doi.org/ 10.1029/JC080i003p00320.

Chereskin, T. K., K. A. Donohue, D. R. Watts, K. L. Tracey, Y. L. Firing, and A. L. Cutting, 2009: Strong bottom currents and cyclogenesis in Drake Passage. Geophys. Res. Lett., 36, L23602, https://doi.org/10.1029/2009GL040940. 
Cunningham, S. A., S. G. Alderson, B. A. King, and M. A. Brandon, 2003: Transport and variability of the Antarctic Circumpolar Current in Drake Passage. J. Geophys. Res., 108, 8084, https://doi.org/10.1029/2001JC001147.

de Lavergne, C., G. Madec, J. L. Sommer, A. J. G. Nurser, and A. C. Naveira Garbato, 2016: The impact of a variable mixing efficiency on the abyssal overturning. J. Phys. Oceanogr., 46, 663-681, https://doi.org/10.1175/JPO-D-14-0259.1.

Dossmann, Y., M. G. Rosevear, R. W. Griffiths, A. M. Hogg, G. O. Hughes, and M. Copeland, 2016: Experiments with mixing in stratified flow over a topographic ridge. J. Geophys. Res. Oceans, 121, 6961-6977, https://doi.org/10.1002/2016JC011990.

Gayen, B., G. O. Hughes, and R. W. Griffiths, 2013: Completing the mechanical energy pathways in turbulent RayleighBenard convection. Phys. Rev. Lett., 111, 124301, https:// doi.org/10.1103/PhysRevLett.111.124301.

Goff, J. A., and T. H. Jordan, 1988: Stochastic modeling of seafloor morphology: Inversion of sea beam data for second-order statistics. J. Geophys. Res., 93, 13 589-13 608, https://doi.org/ 10.1029/JB093iB11p13589.

_ , Y. Ma, A. Shah, J. R. Cochran, and J. C. Sempere, 1997: Stochastic analysis of seafloor morphology on the flank of the Southeast Indian Ridge: The influence of ridge morphology on the formation of abyssal hills. J. Geophys. Res., 102, $15521-$ 15 534, https://doi.org/10.1029/97JB00781.

Klymak, J., 2018: Nonpropagating form drag and turbulence due to stratified flow over large-scale abyssal hill topography. J. Phys. Oceanogr., 48, 2383-2395, https://doi.org/ 10.1175/JPO-D-17-0225.1.

Kunze, E., E. Firing, J. M. Hummon, T. K. Chereskin, and A. M. Thurnherr, 2006: Global abyssal mixing inferred from lowered ADCP shear and CTD strain profiles. J. Phys. Oceanogr., 36, 1553-1576, https://doi.org/10.1175/JPO2926.1.

Legg, S., and J. Klymak, 2008: Internal hydraulic jumps and overturning generated by tidal flow over a tall steep ridge. J. Phys. Oceanogr., 38, 1949-1964, https://doi.org/10.1175/2008JPO3777.1.

Marshall, J., A. Adcroft, C. Hill, L. Perelman, and C. Heisey, 1997: A finite-volume, incompressible Navier-Stokes model for studies of the ocean on parallel computers. J. Geophys. Res., 102, 5753-5766, https://doi.org/10.1029/96JC02775.

Melet, A., R. Hallberg, S. Legg, and M. Nikurashin, 2014: Sensitivity of the ocean state to lee wave-driven mixing. J. Phys. Oceanogr., 44, 900-921, https://doi.org/10.1175/JPO-D-13-072.1.

,,-- A. Adcroft, M. Nikurashin, and S. Legg, 2015: Energy flux into internal lee waves: Sensitivity to future climate changes using linear theory and a climate model. J. Climate, 28, 2365-2384, https://doi.org/10.1175/JCLI-D-14-00432.1.

Meyer, A., K. L. Polzin, B. M. Sloyan, and H. E. Phillips, 2016: Internal waves and mixing near the Kerguelen Plateau. J. Phys. Oceanogr., 46, 417-437, https://doi.org/10.1175/JPO-D-15-0055.1.

Munk, W., and C. Wunsch, 1998: Abyssal recipes II: Energetics of tidal and wind mixing. Deep-Sea Res., 45, 1977-2010, https:// doi.org/10.1016/S0967-0637(98)00070-3.

Naveira Garabato, A., K. L. Polzin, B. A. King, K. J. Heywood, and M. Visbeck, 2004: Widespread intense turbulent mixing in the Southern Ocean. Science, 303, 210-213, https:// doi.org/10.1126/science.1090929.

Nikurashin, M., and R. Ferrari, 2010a: Radiation and dissipation of internal waves generated by geostrophic flows impinging on small-scale topography: Theory. J. Phys. Oceanogr., 40, 10551074, https://doi.org/10.1175/2009JPO4199.1. $\longrightarrow$, and $\longrightarrow$ 2010b: Radiation and dissipation of internal waves generated by geostrophic flows impinging on small-scale topography: Application to the Southern Ocean. J. Phys. Oceanogr., 40, 2025-2042, https://doi.org/10.1175/2010JPO4315.1.

_- and _- 2011: Global energy conversion rate from geostrophic flows into internal lee waves in the deep ocean. Geophys. Res. Lett., 38, L08610, https://doi.org/10.1029/ 2011 GL046576.

— internal waves in the deep ocean. Geophys. Res. Lett., 40, 3133-3137, https://doi.org/10.1002/grl.50542.

,,-- N. Grisouard, and K. Polzin, 2014: The impact of finiteamplitude bottom topography on internal wave generation in the Southern Ocean. J. Phys. Oceanogr., 44, 2938-2950, https://doi.org/10.1175/JPO-D-13-0201.1.

Richet, O., C. Muller, and J. M. Chomaz, 2017: Impact of a mean current on the internal tide energy dissipation at the critical latitude. J. Phys. Oceanogr., 47, 1457-1471, https://doi.org/ 10.1175/JPO-D-16-0197.1.

Roquet, F., C. Wunsch, and G. Madee, 2011: On the patterns of wind-power input to the ocean circulation. J. Phys. Oceanogr., 41, 2328-2342, https://doi.org/10.1175/JPO-D-11-024.1.

Scott, R. B., J. A. Goff, A. C. Naveira Garabato, and A. J. G. Nurser, 2011: Global rate and spectral characteristics of internal gravity wave generation by geostrophic flow over topography. J. Geophys. Res., 116, C09029, https://doi.org/ 10.1029/2011JC007005.

Shakespeare, C. J., and A. M. Hogg, 2017: The viscous lee wave problem and its implications for ocean modelling. Ocean Modell., 113, 22-29, https://doi.org/10.1016/j.ocemod.2017.03.006.

Sheen, K. L., and Coauthors, 2013: Rates and mechanisms of turbulent dissipation and mixing in the Southern Ocean: Results from the Diapycnal and Isopycnal Mixing Experiment in the Southern Ocean (DIMES). J. Geophys. Res. Oceans, 118, 2774-2792, https://doi.org/10.1002/jgrc.20217.

St. Laurent, L. C., A. C. Naveira Garabato, J. Ledwell, A. M. Thurnherr, J. M. Toole, and A. J. Watson, 2012: Turbulence and diapycnal mixing in Drake Passage. J. Phys. Oceanogr., 42, 2143-2152, https://doi.org/10.1175/JPO-D-12-027.1.

Trossman, D. S., S. Waterman, K. L. Polzin, B. K. Arbic, S. T. Garner, A. C. Naveira Garabato, and K. L. Sheen, 2015: Internal lee wave closures: Parameter sensitivity and comparison to observations. J. Geophys. Res. Oceans, 120, 7997-8019, https://doi.org/10.1002/2015JC010892.

Waterhouse, A. F., and Coauthors, 2014: Global patterns of diapycnal mixing from measurements of the turbulent dissipation rate. J. Phys. Oceanogr., 44, 1854-1872, https:// doi.org/10.1175/JPO-D-13-0104.1.

Waterman, S., A. Naveira Garabato, and K. Polzin, 2013: Internal waves and turbulence in the Antarctic Circumpolar Current. J. Phys. Oceanogr., 43, 259-282, https://doi.org/10.1175/JPO-D-11-0194.1.

, K. Polzin, A. Naveira Garabato, K. Shen, and A. Forryan, 2014: Suppression of internal wave breaking in the Antarctic Circumpolar Current near topography. J. Phys. Oceanogr., 44, 1466-1492, https://doi.org/10.1175/JPO-D-12-0154.1.

Wright, C. J., R. B. Scott, P. Ailliot, and D. Furnival, 2014: Lee wave generation rates in the deep ocean. Geophys. Res. Lett., 41, 2434-2440, https://doi.org/10.1002/2013GL059087.

Yang, L., M. Nikurashin, A. M. Hogg, and B. Sloyan, 2018: Energy loss from transient eddies due to lee wave generation in the Southern Ocean. J. Phys. Oceanogr., 48, 2867-2885, https:// doi.org/10.1175/JPO-D-18-0077.1. 